\title{
Efficient Transport of Voice over IP over Cellular links
}

\author{
Lars- $\AA$ ke Larzon ${ }^{1}$, Hans Hannu ${ }^{2}$, Lars-Erik Jonsson ${ }^{2}$, Krister Svanbro ${ }^{2}$ \\ ${ }^{1}$ Luleå University of Technology, Sweden \\ ${ }^{2}$ Ericsson Research
}

\begin{abstract}
Internet and cellular technologies are merging to decrease transport costs and enable IP-based applications to hundreds of millions of cellular users. Real time voice services will continue to be dominant in future cellular systems and to reduce system costs it is desirable to base all services, including voice, on IP. However, the radio spectrum must be efficiently used to provide mass-market services at reasonable prices, but Internet protocol headers are large in size. Moreover, data discarding policies are poorly suited to the error-proneness of cellular links.

Protocol overhead for IP-based voice services can be reduced by compressing headers, e.g. with the Compressed Real Time Protocol (CRTP). CRTP, however, does not perform well over cellular links since it is not robust in the face of errors. ROCCO, a header compression scheme suitable for cellular systems, delivers more packets to the user than CRTP at realistic error rates with a better average compression ratio. Voice services in cellular systems should also be able to use damaged speech data. This can be done using the UDP Lite protocol which provides a more flexible checksumming policy that enables delivery of data possibly usable to the application.

This paper shows that CRTP/UDP fails to deliver sufficient service quality at the error rates of a cost-efficient cellular system and that ROCCO combined with UDP Lite can provide an IP-based voice service over such cellular systems.
\end{abstract}

\section{INTRODUCTION}

With the advent of IP telephony running atop cellular networks, new problems arise for the traditional Internet protocols[1], [2], [3]. Network and transport protocol support for voice over IP in cellular systems has not yet been fully developed and optimized. Traditional TCP/IP support cannot be used because TCP can never provide usable lower delay bounds for voice packets. UDP/IP support, especially in IPv6[4], provides a service that can throw away whole voice packets when bit errors are detected. Cellular networks make this last problem significant since bit errors occur frequently. The checksumming policy in UDP makes the problem even worse, since speech codecs designed for use in cellular environments usually can handle damaged data to some extent.

With this in mind, the UDP Lite protocol was designed.
It provides the option to define a part of each datagram as insensitive to errors. Errors in the insensitive part will be ignored by the checksum mechanisms, and the decision whether a damaged datagram is usable or not is moved from the transport protocol to the application itself. Even with this extra flexibility, the UDP Lite protocol is still compatible with classic UDP.

Another problem is that headers are getting larger for network and transport protocols, especially for IPv6, and channel bandwidth can be wasted with header information. Since IP telephony services typically use the RTP protocol, the IP/UDP/RTP headers can be compressed from 40 or 60 octets down to as little as 2 octets by using CRTP [5]. However, IP telephony in cellular systems is hard to realize with satisfactory speech quality using CRTP due to the error-proneness and long round trip times of cellular links[6].

ROCCO (Robust Checksum-based Compression)[7] is a header compression algorithm which solves this problem by making the IP/UDP/RTP header compression more tolerant of loss of compressed packets. Headers are compressed down to as little as 1 octet per packet. This level of compression is possible since ROCCO supports compression profiles which use knowledge about packet contents for different types of traffic. Comparing ROCCO to CRTP over links in a cellular system shows that CRTP loses 6 times more packets than ROCCO, causing decreased speech quality [8].

By combining ROCCO with UDP Lite, it becomes possible to implement cost-efficient IP telephony services in cellular systems where this previously could not be done with satisfactory speech quality. Circuit-switched telephony services can be replaced with packet switched, which gives several benefits. Apart from the advantage of basing communication on the widely deployed Internet Protocol suite, the system cost is reduced. The need for solutions based on service proxies, portals or alternate protocol stacks will also decrease.

In this paper, CRTP and ROCCO have been simulated 
over realistic WCDMA links with both UDP and UDP Lite as transport protocol. The results show that UDP Lite together with ROCCO can cope with bit error rates up to about $10^{-3}$ with a packet loss rate of less than $1 \%$, which would give sufficient speech quality [9]. Achieving the same quality when using UDP and CRTP requires a bit error rate lower than $10^{-4}$. This is expensive to realize in a cellular system since it would consume too much of the expensive radio spectrum and reduce the total cellular system capacity.

This paper is organized as follows. Section 2 describes the problems with IP in cellular systems in more detail. The ROCCO compression scheme and UDP Lite protocol are presented briefly in section 3 . Section 4 describes simulations of WCDMA links, together with discussions about the results. Finally, the conclusions are presented in section 5 .

\section{IP TELEPHONY IN CELLULAR SYSTEMS}

\section{A. Background}

The widespread and strong growth of the Internet has created a mass market for all kinds of applications, from information applications to multimedia services. In a similar way, the strong growth and evolution of cellular technologies have provided users with true mobility. Communication is nowadays always possible regardless of location. The combination of the service flexibility in IP and the mobility of cellular technologies thus seems very attractive.

The telecom industry is currently starting to embrace the IP protocol suite as the choice of communication framework for the future. To ensure application independence and to decrease transport and switching costs it is attractive to go IP all the way over the air interface to the end user equipment, i.e., to not terminate Internet protocols before the air interface. IP all the way enables service flexibility, i.e., there are no dependencies between applications and the radio access network. A base is created where many players can participate and develop new applications. This should be compared with present-day cellular services, which are vertically integrated and optimized - resulting in very high radio performance but low service flexibility. Cellular access networks have been optimized for quality and spectrum efficiency, resulting in a highly efficient circuit switched voice service. The requirements in terms of spectrum efficiency and voice quality for voice over IP services in future all IP cellular systems will reasonably be similar to current requirements on the circuit switched service.

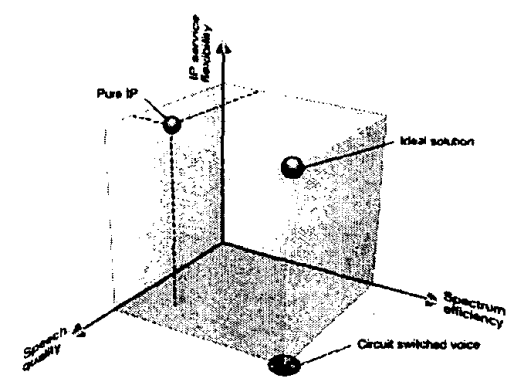

Fig. 1. The challenge cube

The demand for service flexibility adds a new parameter, as illustrated by Figure 1. For mass-market services like cellular telephony, the main challenge is to achieve service quality and spectrum efficiency. To date, all cellular systems that provide voice services have been optimized in a two-dimensional space whose $\mathrm{X}$ - and $\mathrm{Y}$-axes are speech quality and spectrum efficiency. Now, a third dimension is being added in the form of service flexibility. The goal of reaching the ideal solution with sufficient speech quality and spectrum efficiency together with full IP service flexibility introduces new challenges when realizing voice over IP over cellular. These challenges become even bigger when the harsh radio environment with relatively large delays and a high bit error rate (BER) is combined with requirements on high system capacity and good service quality [9]. The main problems to overcome when realizing efficient voice services over cellular systems are:

- How to make efficient use of the scarce radio spectrum. In a cellular system for mass usage, the shortage of radio spectrum is always the limiting resource. To allow cheap mass-market services, many users must share the scarce radio spectrum while still getting sufficient quality. This calls for robust and low-overhead solutions. In cellular systems, the costs of base stations and radio spectrum are high. This implies a tight reuse of the radio spectrum while keeping down the number of base stations to reduce costs. This will result in an increase in interference and thus, a lowered carrier to interference ratio.

- The harsh radio conditions.

Even with complex signaling processing, the radio environment is harsh. Multi-path fading, shadow fading and time dispersion increase the probability of bit errors on 
cellular links. These phenomena become even more problematic when the number of users in a system is maximized, as for mass-market service voice. The interference level increases and the carrier to interference ratio decreases. An illustrative example is a situation where a user enters the subway or a basement during a call. The change of surrounding environment will cause big changes in received signal strength and bit error rates may thus vary heavily.

- The relative large delays.

Sophisticated channel encoding and interleaving to combat bit errors cause delays. Additional delay is also caused in internal nodes managing mobility in the cellular network. Hence, a cellular link may be subject to a one-way delay of $100 \mathrm{~ms}$. It is difficult to estimate delays in future cellular systems, but in e.g. the GSM system, the minimum one-way delay is $80 \mathrm{~ms}$.

Further, radio bearers (the point-to-point connections between mobile terminals and the base station) suitable to the traffic they are carrying have to be selected. Overprovisioning wastes the scarce spectrum while underprovisioning gives too bad service quality.

When realizing IP-based voice services in cellular systems, which meet the spectrum efficiency and quality requirements of present-day cellular systems, new challenges are encountered.

- The relatively large IP header overhead has to be reduced.

A major problem with voice over IP are the large headers of the protocols used when sending speech data. An IPv4 packet with speech data will have an IP header, a UDP header, and an RTP header making a total of $20+8+12=40$ octets. With IPv6, the IP header is 40 octets for a total of 60 octets. The size of the speech data depends on the codec, but can be 15-30 octets. These figures present a major reason for not using IP over the air interface if the overhead cannot be reduced, e.g. by using header compression. However, the error-proneness and the large delays of cellular links impose new requirements on header compression schemes. A viable header compression scheme for usage in cellular systems must produce very small headers to enable efficient usage of the scarce spectrum while still being robust to the error patterns.

- How to make use of damaged packets.

To allow efficient usage of the radio link and to increase the speech quality, all packets that have been transmitted over the air-interface must be made available to the application. Typically a codec used for voice services in cellular systems is capable of handling errors in speech data to some extent, but usage of IP-based solutions will prevent such data from reaching the application. A failing checksum in the transport layer will cause possibly usable packets to be discarded.

- Radio bearers suitable to the traffic they are carrying must be used.

To realize spectrum efficient solutions, radio bearers must be tailor-suited to the services they are carrying. This requires that information about the service is available. Today's QoS mechanisms in the TCP/IP protocol suite, i.e. IntServ and DiffServ, do not provide sufficient information. Additional mechanisms for providing sufficient information must somehow be added.

\section{ENABling IP solutions}

In this section, we present solutions to the problems with overhead and discarding of possibly usable packets when using IP in cellular systems.

\section{A. Solving the overhead problem}

To make an IP-based solution for pure speech economically feasible as a replacement for traditional solutions used in cellular systems, the overhead introduced by the Internet protocols must be significantly reduced over cellular links. This can be and have successfully been done with various header compression methods [10], [11], [5], applied on a per-hop basis. Header compression is possible thanks to a high degree of redundancy between header fields within each packet and, especially, between consecutive packets. Header compression methods maintain, at both sides of a link, a shared state called context, including essentially the uncompressed version of the last header sent over the link. Compression and decompression are done relative to the context, and only unpredictable changes are transferred. While static header information needs to be transmitted only once, dynamic information can be transmitted as changes from previous packet using only a few bits. When packets are lost over the link, the decompressor context will be brought out of sync and decompression of subsequent headers will fail. Thus, in addition to methods for initializing and updating the context, header compression schemes must also have mechanisms for detection of incorrect decompression and ways to repair an invalidated decompressor context.

Today, there exist several header compression schemes of which one, CRTP [5], is suitable for real-time services 
using the Real-time Transport Protocol, RTP [12]. CRTP can compress the 40 octet IPv4/UDP/RTP headers to a minimum of 2 octets when the UDP checksum is disabled. ${ }^{1}$ To repair an invalidated context, CRTP makes use of explicit signaling messages by which the decompressor requests a context update from the compressor. The link round trip time will thus affect the time it takes to update the decompressor context. While the context is invalid, all packets received by the decompressor will be discarded, meaning that a long link round trip time will generate a large number of lost packets. On lossy links with long round trip times, such as most cellular links, CRTP does not perform well. This has been studied in [13], where it was found that the compression ratio of CRTP is satisfactory but that the packet loss rate after decompression at the receiver is too high over such links.

It is clear that CRTP alone is not a viable header compression scheme for cellular links. For such environments, a more robust scheme that can tolerate a higher error rate on the link, while being at least as efficient as CRTP, is needed. It is of course important that the decompressor always produce correctly reconstructed headers and packets.

\section{B. Robust Checksum-based header Compression (ROCCO)}

To fulfill the requirements stated above, a new header compression scheme has been developed which is adaptable both to the characteristics of the packet streams it compresses and to the characteristics of the link over which it is used. The scheme is called ROCCO (Robust Checksum-based header Compression)[7] and has, thanks to its adaptability, exhibited excellent performance both in terms of compression efficiency and robustness against packet loss on the link[8].

ROCCO is heavily geared towards local decompressor repair of the context. A CRC covering the original, uncompressed header is included in the compressed header, providing a reliable way to verify the correctness of the reconstructed header, as shown in figure 2.

With this mechanism provided, the decompressor can perform several reconstruction attempts to find the correct header. This is an important possibility when packet loss on the link occurs, causing the decompressor context to be out of sync. The principle is illustrated in figure 3 , showing the way the decompressor performs several reconstruction attempts, if needed, to find the correct

${ }^{1}$ The UDP checksum can be disabled in IPv4, but not in IPv6.

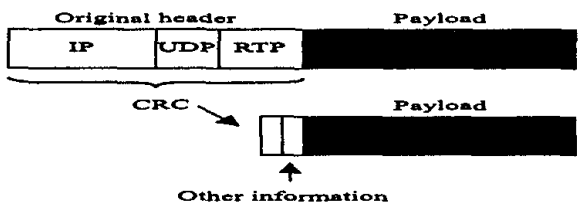

Fig. 2. ROCCO Compression principle

header. Only time and computational complexity bound the number of possible reconstruction attempts. Further, the mechanisms in ROCCO with an internal CRC practically eliminate the possibility of incorrect header reconstruction, e.g., due to undetected bit errors from lower layers.

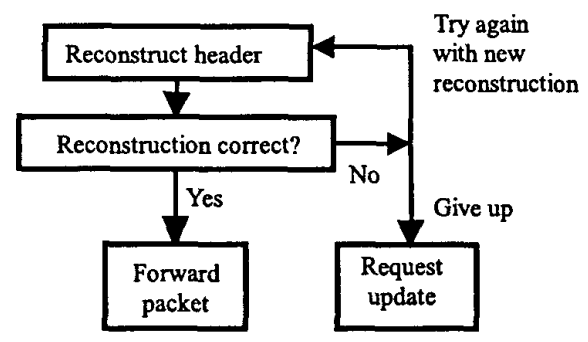

Fig. 3. ROCCO decompression principle

The adaptation of ROCCO to both packet stream and link characteristics is possible through a concept built upon compression profiles. Today there exist various compression profiles for voice and video streams that are suited for typical cellular link properties. However, more generalized profiles will be developed for all kind of applications, but with slightly reduced compression performance. Today, ROCCO profiles for voice over IP exist, which can compress IP/UDP/RTP headers down to minimum sizes of 1-2 octets, with the average sizes being only slightly larger.

It has been shown that ROCCO significantly reduces the negative effect on header compression performance that are otherwise caused by high packet loss and long round trip times. Still, ROCCO compresses the headers as well as or better than CRTP and provides a reliable protection against generation of erroneous headers. 


\section{UDP Lite}

ROCCO addresses the problems with expensive bandwidth, but IP packets which have made it across a cellular link might be discarded by UDP due to a failing checksum. If damaged data packets are usable by the codec, it is not desirable that they should be discarded by the transport layer. In cellular phone systems this is solved by allowing damaged data to reach the codec as such data often turns out to be usable. When a damaged speech packet reaches a cellular speech decoder, parts of the data are used together with previous speech packets to synthesize speech and conceal the error.

Enabling delivery of damaged packets to the application can be achieved in IPv4[1] by disabling the UDP checksum, but doing so will remove the verification of protocol headers for UDP and RTP as well. Therefore it is usually undesirable to disable the UDP checksum. Moreover, if IPv6 is used to provide Internet connectivity the UDP checksum is mandatory and cannot be disabled. This will cause a high percentage of potentially usable packets to be discarded by the transport layer.
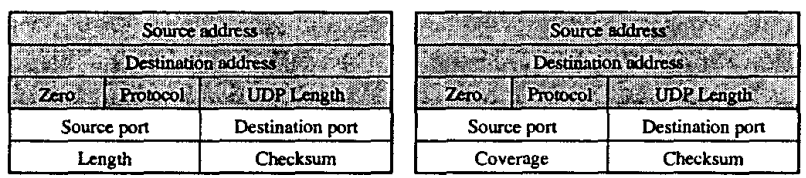

Fig. 4. The UDP and UDP Lite headers, respectively

The UDP Lite protocol [14] solves this problem by replacing the redundant datagram length information in the UDP header with a Coverage value as shown in figure 4. This modification will not disturb intermediate nodes along a path since the length field in the UDP header is used only at the endpoints.

Only the first Coverage octets of the UDP Lite datagram are verified by the checksum. If the value is equal to the packet length, the UDP Lite datagram is identical to a classic UDP datagram ${ }^{2}$. By setting the coverage to less than the datagram length, the datagram is divided into one sensitive and one insensitive part. Errors in the sensitive part will result in discarded datagrams, while errors in the insensitive part are ignored.

UDP Lite provides the flexibility for applications to move the decision whether damaged data is usable from

\footnotetext{
${ }^{2}$ This assumes there is no padding in the IP payload after the UDP datagram. Such padding is rare and usually unneeded.
}

the transport protocol to the application itself. In situations where it is essential not to discard usable data, this flexibility is important. While providing this extra flexibility, it is fully compatible with classic UDP. The simple design of the protocol makes it easy to implement as an extension or derivate of almost any existing implementations of classic UDP.

\section{Performance measurements}

\section{A. Setup}

In order to more closely investigate the performance differences between CRTP/ROCCO and UDP/UDP Lite, detailed simulations including a $3 \mathrm{G}[15]$ cellular link have been made according to the scenario shown in figure 5. A source generates speech packets, which are carried through the network by IP. The packets consist of the IP/UDP/RTP headers and the speech data. The network, symbolized by a cloud in figure 5 , corresponds to various wired and/or wireless links. The last link on the path to the receiver is a cellular link, over which the IP/UDP/RTP headers are compressed. ROCCO and CRTP are the two examined header compression algorithms. For ROCCO, a profile with a minimum header size of 2 octets is evaluated.

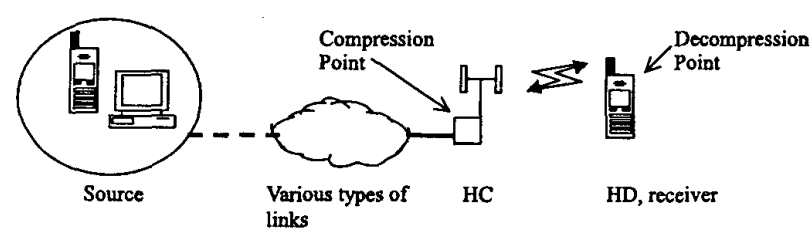

Fig. 5. Scenario for link level simulations

The source in the simulations has the characteristics of an AMR speech codec running in $12.2 \mathrm{kbps}$ mode with silence suppression. The speech and silence intervals are both exponentially distributed with a mean value of one second. The packet loss rate in the cloud is uniformly distributed with a probability of $0.5 \%$. No reordering of packets takes place.

Header compression algorithms need mechanisms to identify different packet (header) types. CRTP needs to have the packet type identification provided by the link layer whereas ROCCO has the packet type identification integrated. Hence, one octet of link layer overhead is added to the compressed packets for the CRTP case $^{3}$. For

\footnotetext{
${ }^{3}$ This extra octet is not included when calculating the average
} 
the ROCCO case, the link layer is transparent. It is assumed that the lower layers of the cellular link can provide the header compression algorithms with an indication if there are any bit errors in the compressed header part of the packet. In the third generation of cellular systems, WCDMA is one of the carrier techniques, which is why a WCDMA link is used as the cellular link. See table I for a more detailed description of the link properties. The reported BER is that seen by the link layer, i.e., the BER visible just below the header compression layer. For both ROCCO and CRTP, a reverse channel is needed. The reverse channel in these simulations never damages the context request messages 4 and the round trip time between the compression and decompression points is set to $120 \mathrm{~ms}$, which is a realistic round trip time in a cellular system[9].

\begin{tabular}{|c|c|}
\hline Link type & WCDMA \\
\hline Interference & Gaussian white noise \\
\hline Coding & $1 / 3$ Convolutional \\
\hline Fading model & Indoor to Outdoor pedestrian \\
\hline Mobile speed & $3 \mathrm{~km} / \mathrm{h}$ \\
\hline Spreading factor & 128 \\
\hline Power control & Ideal fast \\
\hline
\end{tabular}

TABLE I

Properties of the Simulated LiNK

\begin{tabular}{|c|c|c|c|c|}
\hline Case & CRTP & ROCCO & Classic UDP & UDP Lite \\
\hline \hline 1 & $\mathrm{X}$ & & $\mathrm{X}$ & \\
\hline 2 & $\mathrm{X}$ & & & $\mathrm{X}$ \\
\hline 3 & & $\mathrm{X}$ & $\mathrm{X}$ & \\
\hline 4 & & $\mathrm{X}$ & & $\mathrm{X}$ \\
\hline
\end{tabular}

TABLE II

Simulation CASES

In this paper, four cases are studied, as shown in table header size.
${ }^{4}$ The error-free property of the back channel favors CRTP, since
CRTP relies heavily on the reverse channel for error recovery.
II. When simulating the usage of UDP, the UDP checksum is enabled. For the UDP Lite cases, the UDP Lite checksum covers only the IP/UDP Lite/RTP headers. A packet is considered lost if it is not passed up the application, i.e., to the speech codec. This can occur for four different reasons in these simulations:

1. A bit error has occurred in the compressed header.

2. A bit error has occurred in the link layer checksum or packet type identification bits.

3. The decompressor has an invalid context and cannot decompress any received compressed header ${ }^{5}$.

4. In the case with classic UDP a packet is also lost if it has any bit errors in the payload (speech data). In the cases with UDP Lite a packet with errors in the payload is not regarded as lost as long as it is considered 'ok' by the header compression scheme. This is because the UDP Lite checksum covers only the headers and not the speech data.

\section{B. Results}

Figure 6 shows the packet loss distribution for ROCCO and CRTP, with classic UDP and UDP Lite. The difference between the two header compression schemes becomes evident from this figure. The majority of the packet losses with CRTP are caused by its context repair mechanism, which is heavily dependent on the round trip time. ROCCO does not suffer from context damage as it can handle consecutive packet losses between the compression and decompression point without losing context synchronization. The choice of transport protocol does not significantly affect the distribution of consecutive packet losses. This is not surprising since it is mainly header compression that can cause consecutive packet losses.

Figure 7 shows the packet loss rate versus channel BER. This figure shows the enhanced robustness of ROCCO compared to CRTP. A packet loss between a CRTP compressor and decompressor results in a burst of additional packet losses due to CRTPs repair mechanism. For CRTP, the packet loss rate becomes unacceptably high at typical cellular BERs. At a BER of $10^{-3}$, the loss rate for CRTP is approximately 8 times higher than for ROCCO. There is also a clear difference in packet loss rate when using classic UDP and UDP Lite. ROCCO with UDP Classic results in a packet loss rate about 3 times higher than for ROCCO with UDP Lite, at a BER of $10^{-3}$. Comparing classic UDP with UDP Lite when using CRTP, the packet

\footnotetext{
${ }^{5}$ This can happen even if the compressed header is error free.
} 

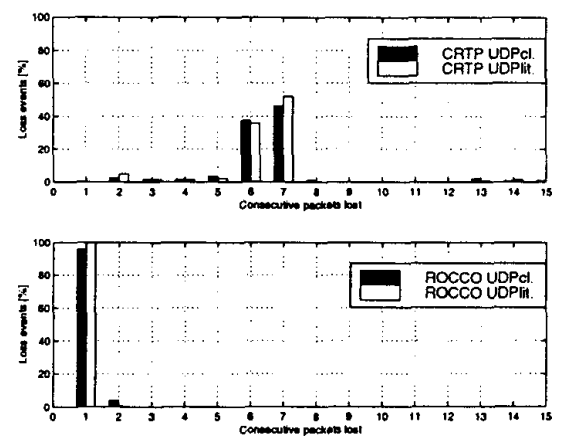

Fig. 6. Packet loss pattern, channel BER: $10^{-3}$

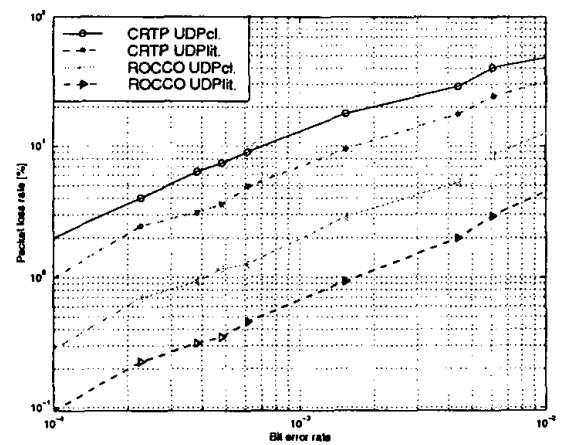

Fig. 7. Packet loss rate versus channel BER

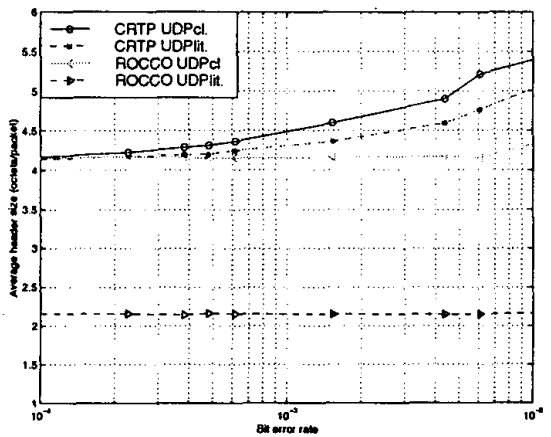

Fig. 8. Average header size versus channel BER

loss rate is about twice as high with classic UDP. How-

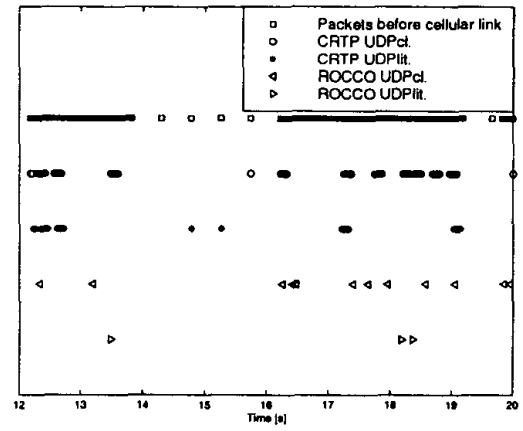

Fig. 9. Packets transmitted and packet loss patterns

ever, the packet loss rate is already too high to achieve acceptable speech quality.

Figure 8 shows the average header size plotted against channel BER. The observed difference between CRTP and ROCCO occurs because the latter tolerates loss of a few consecutive packets before it needs a context update packet, while CRTP needs a context update for each loss. Since the context is rarely brought out of sync for $\mathrm{ROCCO}$, the average header size is slightly larger than the minimum header size. For CRTP, which needs the context update packets ( 17 octets) more often, the average compressed header increases as the link BER increases. With classic UDP, the checksum is mandatory and thus the compressed header size is increased by 2 octets.

The ROCCO header includes a CRC covering the uncompressed original headers. When the UDP Lite checksum covers only the headers (as in these simulations), the same data is protected by both UDP Lite and ROCCO. Since ROCCO provides stronger error protection than UDP Lite, the UDP Lite checksum does not have to be transmitted over the link. At the decompressor, the checksum is reconstructed if the ROCCO CRC succeeds. Hence, the size of compressed ROCCO headers averages just above the minimum header size when using UDP Lite. When using CRTP, the checksum must be included in each compressed packet.

Figure 9 shows the packets before compression and packet loss patterns over the link. At the top of the figure the packets are shown before they have passed the cellular link. The other lines show packet losses for each simulated case. With CRTP we see the characteristic long loss events due to its context repair mechanism. For ROCCO 
on the other hand there are almost only single packet losses, because of its robustness against packet losses. The increase in loss can also be seen when using classic UDP instead of UDP Lite.

\section{Conclusions}

In order to provide cost-efficient solutions for data services in cellular networks, it is desirable to base these on the Internet protocols. This could reduce system costs, especially when new services are introduced, since standard IP switches and routers can be used.

When dealing with scarce bandwidth resources, such as in a cellular network, the protocol overhead should be kept to a minimum. The long round trip times and errorproneness of cellular links imply that real time services must not depend on retransmission of lost or damaged data, which are the main reasons for the problems with CRTP in cellular networks. Finally, data which has traversed a cellular link should not be discarded if usable. Since the decision whether data is usable or not is best made by the application itself, lower layers in the protocol stack must provide enough flexibility to avoid undesired discarding of packets.

The ROCCO header compression algorithm deals with the overhead and round-trip time problems. Protocol headers can be compressed down to a few octets. Profiles for different traffic types are used for different traffic types to provide a higher compression level. Consecutive packet losses will not result in context loss as easily as when using CRTP.

The UDP Lite protocol provides the flexibility for applications to move the decision whether a packet should be discarded due to bit errors from the transport layer to the application itself. This is especially useful for realtime services that often have the capability to handle a damaged payload to some extent.

Simulations show that at realistic bit error rates, the packet loss rate of CRTP is 6 times higher compared to ROCCO when using classic UDP. Using UDP Lite instead of classic UDP together with CRTP will deliver about twice as many packets to the applications. When comparing CRTP/classic UDP to ROCCO/UDP Lite, the ROCCO/UDP Lite solution will deliver at least an order of magnitude more packets to the applications.

By combining ROCCO with UDP Lite, it is possible to use IP-based, packet-switched voice services in cellular systems with sufficient speech quality and spectrum efficiency. Internet protocols can be used to provide global connectivity as well as telephony services for mobile devices.

\section{REFERENCES}

[1] Jon Postel, "Internet Protocol," Request For Comments RFC 791, DARPA, September 1981.

[2] Jon Postel, "Tranmission Control Protocol," Request For Comments RFC 793, DARPA, September 1981.

[3] Jon Postel, "User Datagram Protocol," Request for Comments RFC 768, ISI, August 1980.

[4] Stephen Deering and Robert Hinden, "Internet Protocol, Version 6 (IPv6)," Request for Comments RFC 2460, Internet Engineering Task Force, December 1998.

[5] Stephen Casner and Van Jacobson, "Compressing IP/UDP/RTP Headers for Low-Speed Serial Links," Request for Comments RFC 2508, Internet Engineering Task Force, February 1999

[6] Mikael Degermark, Hans Hannu, Lars-Erik Jonsson, and Krister Svanbro, "Evaluation of CRTP performance over cellular radio links," to appear in IEEE Personal Communications specil issue on IP-based Mobile Telecommunications Networks, 2000.

(7) Lars-Erik Jonsson, Mikael Degermark, Hans Hannu, and Krister Svanbro, "Robust checksum-based header compression(ROCCO)," Internet-Draft (work in progress) draft-ietfrohc-rtp-rocco-01.txt, Ericsson Research, June 2000.

[8] Krister Svanbro, Hans Hannu, Lars-Erik Jonsson, and Mikael Degermark, "Wireless real time IP-services enabled by header compression," to appear in proceedings of IEEE VTC2000, May 2000.

[9] Lars Westberg and Morgan Lindquist, "Realtime Traffic over Cellular Access Networks," Internet-Draft (work in progress) draft-westberg-realtime-cellular-02.txt, Ericsson Research, May 2000.

[10] Van Jacobson, "Compressing TCP/IP headers for low-speed serial links," Request For Comments RFC 1144, LBL, February 1990.

[11] Mikael Degermark, Björn Nordgren, and Stephen Pink, "IP Header Compression," Request for Comments RFC 2507, Luleå University of Technology, February 1999.

[12] Henning Schularinne, Stephen Casner, Ron Frederick, and Van Jacobson, "RTP: A Transport protocol for Real-Time Applications," Request for Comments RFC 1889, Internet Engineering Task Force, January 1996.

[13] Mikael Degermark, Hans Hannu, Lars-Erik Jonsson, and Krister Svanbro, "CRTP over cellular radio links," Internet-Draft (work in progress) draft-degermark-crtp-cellular-01.txt, Luleå University of Technology, December 1999.

[14] Lars-Åke Larzon, Mikael Degermark, and Stephen Pink, "The UDP Lite Protocol," Internet-Draft (work in progress) draftlarzon-udplite-03.txt, Luleå University of Technology, July 2000.

[15] "Third generation partnership project (3GPP) homepage: http://www.3gpp.org/,", 